\title{
Quaderni
}

QUADERNI Communication, technologies, pouvoir

\section{Comment évaluer et réguler la performance en matière de qualité de la prise en charge des malades?}

\section{Étienne Minvielle}

\section{(2) OpenEdition \\ Journals \\ Édition électronique \\ URL : http://journals.openedition.org/quaderni/747 \\ DOI : $10.4000 /$ quaderni.747 \\ ISSN : 2105-2956 \\ Éditeur \\ Les éditions de la Maison des sciences de l'Homme}

\section{Édition imprimée}

Date de publication : 5 octobre 2013

Pagination : 83-98

Référence électronique

Étienne Minvielle, «Comment évaluer et réguler la performance en matière de qualité de la prise en charge des malades? », Quaderni [En ligne], 82 | Automne 2013, mis en ligne le 05 octobre 2015, consulté le 01 mai 2019. URL : http://journals.openedition.org/quaderni/747 ; DOI : 10.4000/ quaderni.747 


\title{
Politique
}

\section{Comment évaluer et réguler la performance en matière de qualité de la prise en charge des malades?}

Dans le système de santé français, la régulation de la qualité vise à garantir un niveau de qualité égal quel que soit les conditions d'accès. Fort de ce principe de solidarité, le régulateur ne doit pas contribuer à différencier par la qualité, mais au contraire la rendre homogène et subordonner toute concurrence à un objectif de réduction des inégalités.

Avant les années 1990, il était difficile de rattacher un contenu précis à ce principe. La régulation pouvait s'évoquer à travers le respect de normes techniques, ou des décisions de planification sur des activités ciblées. Mais, hormis ces situations, la régulation de la qualité restait limitée : peut-être du fait de la méconnaissance relative du niveau réel de la performance en matière de qualité, sans doute parce que l'opinion publique restait favorable à l'égard du système, probablement à cause de l'autonomie médicale prédominante. $\mathrm{Au}$ fond, rien ne valait mieux que la confiance accordée aux médecins pour assurer la meilleure qualité des soins dans le cadre du colloque singulier.

Les choses ont pourtant changé. La régulation de Minvielle

\author{
Professeur à l'EHESP \\ Directeur de la qualité \\ à l'Institut Gustave Roussy
}

la qualité des soins s'est progressivement constituée comme un objectif à part entière du système de santé. Plusieurs constats ont conduit à cette évolution : variabilité des pratiques, questions de sécurité, mésusage, sur ou sous-consommation de soins, besoin d'aide à la décision devant la multiplicité des connaissances nouvelles. Cette volonté régulatrice a pu aussi contribuer à alimenter un jeu de rôles où l'amélioration de la qualité constitue selon, un garde-fou face à la recherche d'une productivité excessive, ou un alibi face à la contrainte financière qui pèse sur 
les acteurs. Enfin, elle a participé d'une plus grande transparence du système vis-à-vis du public, démarche logique dans un secteur où la régulation est en majeure partie administrée, et donc doit rendre compte de l'emploi des ressources publiques.

L'objectif de cet article est de décrire l'émergence de ce thème de la régulation de la qualité des soins, en se centrant sur les établissements de santé, objets principaux des actions engagées dans le domaine.

Dans un premier temps, un cadrage des concepts de régulation et de qualité est proposé. La seconde partie, consacrée à une mise en perspective historique, dresse un panorama des premières formes de régulation qui se sont développées à partir des années 90. La troisième partie montre comment l'apparition des indicateurs de qualité a introduit de nouvelles formes, en rupture avec les conceptions précédentes. La quatrième s'intéresse aux perspectives. Nous tenterons notamment d'y préciser quel peut être le statut de la régulation de la qualité dans des systèmes professionnels $^{1}$ de ce type : entre contrôle et autonomie encadrée.

\section{La Régulation de la Qualité des soins : Repères}

Peut-être parce qu'ils sont particulièrement utilisés, les concepts de régulation et de qualité des soins restent toujours difficiles à circonscrire. Par ailleurs, l'application de la régulation à la qualité des soins rend l'approche spécifique. L'ensemble appelle à fixer des repères.

\subsection{La qualité des soins : un concept multi- dimensionnel}

Dans les définitions sur le sujet qui sont légions, certaines dimensions s'y retrouvent malgré tout d'une manière constante. Sont ainsi fréquemment distingués des facteurs organisationnels (la coordination), et l'expertise professionnelle (la bonne pratique fondée sur le savoir médical) (Deming, 1986) ${ }^{2}$. Une autre typologie (Donabedian, 1980) ${ }^{3}$, influencée par le souci de l'évaluation à travers des données chiffrées, vise à distinguer la qualité structurelle (un seuil d'activité, par exemple), la qualité des processus ou actions réalisées (une bonne pratique), et la qualité des résultats (la satisfaction du patient).D'autres enfin privilégient des thèmes prioritaires de santé publique : douleur, infections nosocomiales, par exemple ${ }^{4}$.

\subsubsection{Une taxinomie de la qualité}

L'effort de synthèse de ces différents aspects aboutit peu ou prou aux sept dimensions suivantes (cf. Tableau 1) :

- celles en rapport avec la structure : les processus (respect des bonnes pratiques professionnelles), les modes d'organisation de la prise en charge (par exemple, le taux d'annulation d'interventions chirurgicales programmées), les systèmes d'information (par exemple, la tenue du dossier du patient), ou, enfin, l'accès aux soins (délais d'attente mais aussi accessibilité selon la mobilité physique du patient) ;

- celles liées aux résultats : l'efficacité clinique (la mortalité/morbidité), le point de vue de patients (satisfaction, expérience des patients, plaintes), 
la sécurité (les infections nosocomiales et la iatrogénie).

Tableau 1 : Les sept dimensions de la qualité

1. Organisation de la prise en charge.

2. Systèmes d'informations, supports et traitements de données.

3. Pratiques professionnelles.

4. Accessibilité.

5. Risque/Sécurité de la prise en charge.

6. Qualité perçue par le patient.

7. Éfficacité clinique.

Néanmoins, différentes questions subsistent. Un débat récurrent vise notamment à savoir si la sécurité constitue une dimension de la qualité ou un champ autonome. Le développement des indicateurs et la considération de la qualité sous toutes ces facettes, positive et négative, conduisent de plus en plus à ne pas formuler de distinction trop nette et à intégrer l'ensemble dans la gestion globale du risque à l'hôpital.

Une seconde interrogation a trait à la notion d'efficacité clinique. Différents arguments peuvent conduire à percevoir cette dimension comme un champ autonome des résultats (outcomes). La qualité se situant uniquement dans le déroulement de la prise en charge, le résultat qui s'ensuit est en quelque sorte hors du champ. D'autant que la qualité de la prise en charge n'induit pas forcément un bon résultat, du fait de l'aléa lié à l'évolution clinique du malade. Là encore, les différences semblent s'estomper compte tenu des attentes en matière de qualité et de performance.

En revanche, la question de l'accessibilité aux soins déborde le champ de la qualité, même si les deux notions sont intriquées. Ainsi, l'accès d'un point de vue géographique ou du fait du niveau de revenu financier introduisent des questions d'une autre nature relatives à la planification de l'offre de soins et à la justice sociale.

Au total, si la qualité réunit un noyau dur de dimensions, elle ne renvoie pas forcément à une définition univoque. Dès lors, il est plus logique de parler d'une taxinomie de la qualité, et d'être clair sur les dimensions prises ou non en considération (Duhamel, Minvielle, 2009) 5 .

\subsubsection{Liens entre qualité, performance et efficience}

Le positionnement de la qualité par rapport aux concepts de performance et d'efficience n'est pas non plus aisé à saisir en pratique. Dans la plupart des approches, la performance exprime un objectif ou un seuil à atteindre en termes de qualité. Par exemple, on décidera de fixer un seuil de 85 points à atteindre sur les 100 points distribués dans l'indice composite d'actions de lutte contre les infections nosocomiales (indicateur ICALIN utilisé au sein des établissements de santé français depuis 2006) pour être jugé performant en matière de qualité de lutte contre ces infections. Mais dans d'autres cas, la performance se prête à une autre interprétation. Elle contribue généralement à porter un jugement global sur l'activité hospitalière, englobant au passage la qualité. Par exemple, dans le Balance Score Card, modèle à succès en entreprise proposé par Kaplan et Norton (1996) ${ }^{6}$, la qualité est une dimension parmi d'autres, subordonnée à son impact sur l'équilibre financier. En théorie des organisations, l'approche de 
la performance multidimensionnelle développée par Sicotte et al. (1998) ${ }^{7}$ situe la qualité comme une composante de la dimension «production des soins », à côté d'autres dimensions portant sur l'ouverture vers l'extérieur, la définition des objectifs ou le climat organisationnel.

Pour l'économiste, la performance renvoie à une notion d'efficience à la fois technique et allocative. L'efficience technique se conçoit comme l'optimisation d'un résultat à ressources constante (ou à l'inverse comme l'optimisation de l'utilisation des ressources à résultat médical ou sanitaire constant) de la part d'un producteur donné, établissement de santé ou groupe de médecins. L'efficience allocative concerne le système de soins dans son ensemble, incluant les questions de coordination entre hôpital et médecine de ville, ainsi que le système d'information. Dans les deux cas, la performance rejoint la notion d'efficience. Cette approche va au-delà des simples questions d'utilisation des ressources (par exemple, le taux de recours à des médicaments génériques). De surcroît, elle peut permettre de faire le lien entre qualité, efficience et performance : si l'on considère que le résultat de la « fonction d'optimisation » correspond à la qualité du service rendu. L'efficience ou la performance peut dès lors s'entendre comme l'optimisation de la qualité en fonction des ressources allouées, ou comme l'optimisation des ressources allouées à qualité constante. Notons que ces deux déclinaisons ont un sens différent, le second cas mettant plus l'emphase sur le contrôle des coûts que sur la qualité. Soulignons également que la théorie de la valeur telle que décrite par Michael Porter s'inscrit dans cette lecture, prônant l'optimisation des résultats, ou outcomes. au nom de la qualité ${ }^{8}$.

\subsection{Le concept de régulation appliqué à la qualité d'un service à forte composante professionnelle}

Par régulation, on entend habituellement la définition de la mise en œuvre de règles par la puissance publique pour influencer la façon dont les agents conduisent leur activité (Lévêque, 1998) 9 . Mais dans le cas de la qualité des soins, d'autres forces que la puissance publique peuvent contribuer à cette régulation. Par exemple, des associations de malades, des réseaux plus ou moins formalisés entre les professionnels du secteur, des autorités invisibles telles les conventions et les valeurs morales jouent également un rôle, plus ou moins influent. Il convient donc de considérer que l'intervention de la puissance publique n'est pas le seul mécanisme envisageable.

En référence à cette définition extensive, les pratiques de régulation de la qualité dans différents secteurs serviciels à forte composante professionnelle mettent en évidence quatre formes principales (Sappington, 2005) ${ }^{10}$ (cf. Tableau 2) :

- La règlementation (typiquement, les licences d'exercice, les lois, les régimes d'autorisation) à laquelle est associée la régulation par le litige qui repose sur le respect de la règle et l'évitement des sanctions ;

- L'incitation financière (le paiement à la qualité) qui valorise les efforts fournis pour améliorer la qualité ;

- La diffusion publique de l'information (les classements) qui s'attache d'abord à l'opinion de l'autre dans une logique de comparaison; 
- Les règles éthiques (promotion de valeurs) et de l'autodiscipline (l'engagement volontaire à changer les pratiques existantes) qui repose sur le sens du travail bien accompli et un sentiment de responsabilité.

On peut observer que ces formes se distinguent par la nature du levier motivationnel : valeurs professionnelles pour la logique de responsabilisation des acteurs; règles et sanctions pour le registre autoritaire ; information pour la philosophie de comparaison, et argent pour l'incitation financière. Elles ont également des principes, une instrumentation, et des institutions impliquées, distincts comme le montre le Tableau 1 (Pouthier, $2006)^{11}$.

Tableau 2. Différents types de régulation de la qualité d'un service à composante professionnelle

\begin{tabular}{|c|c|c|c|c|}
\hline & $\begin{array}{l}\text { Ethique } \\
\text { Autodiscipline }\end{array}$ & $\begin{array}{l}\text { Règlementation } \\
\text { Judiciarisation }\end{array}$ & $\begin{array}{l}\text { Diffusion } \\
\text { Publique }\end{array}$ & $\begin{array}{l}\text { Incitation } \\
\text { financière }\end{array}$ \\
\hline \multicolumn{5}{|c|}{ PRINCIPES } \\
\hline Logique & Responsabilité & Conformité & ncitation & Incitation \\
\hline Motivation & $\begin{array}{l}\text { Sens de l'engagement et de } \\
\text { la responsabilité }\end{array}$ & $\begin{array}{l}\text { Respect des règles } \\
\text { et évitement de la } \\
\text { sanction }\end{array}$ & $\begin{array}{l}\text { Comparaison } \\
\text { (Image et compé- } \\
\text { titivité) }\end{array}$ & $\begin{array}{l}\text { Reconnaissance de } \\
\text { la valeur des efforts } \\
\text { et Profitabilité }\end{array}$ \\
\hline \multicolumn{5}{|c|}{ INSTRUMENTS } \\
\hline Professionnel & $\begin{array}{l}\text { Normes internalisées, } \\
\text { Code de déontologie, } \\
\text { Culture }\end{array}$ & $\begin{array}{l}\text { Lois, autorisations, } \\
\text { Normes, } \\
\text { Actions judiciaires et } \\
\text { pénalités }\end{array}$ & $\begin{array}{l}\text { Signalling } \\
\text { (marques, labels, } \\
\text { etc.) } \\
\text { Classement }\end{array}$ & Prime au mérite \\
\hline Organisation & $\begin{array}{l}\text { Charte de qualité, Ordre } \\
\text { professionnel }\end{array}$ & & & $\begin{array}{l}\text { Rémunération à la } \\
\text { performance }\end{array}$ \\
\hline
\end{tabular}

Dans le cas de la « qualité des soins », il faut aussi souligner qu'il s'agit d'une activité « multi-services ». Selon les outils les plus répandus d'analyse de l'activité hospitalière, un hôpital peut « produire » entre 600 et 1500 types de prise en charge selon le degré de finesse de la classification. Cette variété de prestation est amplifiée par le fait qu'elle s'applique à des personnes humaines aux caractéristiques sociales et comportementales différentes. Dès lors, il faudrait en toute rigueur envisager une qualité propre à chaque prise en charge. C'est sans doute une des questions les plus centrales de l'évaluation et de la régulation de la qualité appliquée à ce secteur. Il existe un principe d'aller-retour entre une lecture agrégée nécessaire à la régulation et la qualité 
associée à la singularité de chaque patient pris en charge qui introduit une forte tension dans une telle activité de service.

Une interrogation forte réside donc sur la capacité de réguler une qualité de prise en charge si spécifique à chaque malade. Faut-il miser sur l'autonomie des professionnels ou bien sur un contrôle étroit de chaque pratique ? Mais dans ce dernier cas peut-on envisager un dispositif d'évaluation externe qui soit peu coûteux ? Quelles organisations peuvent en assurer l'application ? Et enfin, quels liens peuvent être envisageables avec la régulation des coûts, préoccupation majeure des pouvoirs publics depuis les années 80 ? C'est à toutes ces questions que l'analyse historique vise à apporter un éclairage.

\section{2. À la fin du siècle dernier : une régulation « soft"}

Dans une première phase allant jusqu'à la fin du siècle dernier, la régulation de la qualité des soins hospitaliers a reposé sur des mécanismes de contrôle que l'on peut considérer comme « soft».

\subsection{L'auto-évaluation des pratiques profes- sionnelles, les démarches qualité et l'accréditation}

L'auto-évaluation des pratiques professionnelles est apparue au début des années 1980 au sein des hôpitaux français, pour connaître ensuite un essor important à partir des années 1990, notamment avec la loi hospitalière de 1991 qui pour la première fois a « légitimé » l'évaluation à l'hôpital.

Les médecins, comme les autres membres des équipes soignantes, mènent des actions propres à assurer et à améliorer la qualité de leurs prestations. Certaines sont implicites, d'autres plus systématisées dans le cadre de protocoles. La plupart se réfèrent à la médecine fondée sur les preuves (evidence-based medicine) qui les éclaire sur le contenu des soins, les actes et interventions souhaitables dans des situations-types. Différents travaux se sont inscrits dans cette logique dans le contexte français du développement de recommandations de pratique clinique (Matillon et Durieux, 2000 ${ }^{12}$. En complément, les démarches d'amélioration continue de la qualité apportent une touche organisationnelle, en insistant sur le caractère transversal de la prise en charge du malade, et en empruntant au monde de l'entreprise des concepts de gestion, non d'ailleurs sans certains abus de langage (Minvielle, 2003) ${ }^{13}$.

Enfin pour dynamiser ces actions, les établissements français sont soumis à une procédure d'accréditation. Coordonnée par l'Agence Nationale d'Accréditation (devenue ensuite la Haute Autorité en Santé), cette procédure créée en 1996 consiste dans sa première mouture en une autoévaluation collective, vérifiée par une visite de membres extérieurs pour valider les points forts et faibles identifiés. Après la visite, un rapport est établi qui sert de support à une accréditation conférée pour une durée de quatre ans.

\subsection{Et des interventions réglementaires ciblées}

Parallèlement, une régulation règlementaire est assurée par les pouvoirs publics à travers le respect de normes minimales et ciblées. Par exemple, il s'agit de normes sécuritaires relatives au matériel, au bâtiment, ou à son accessibilité 
aux personnes handicapées. Elles se fondent sur la définition de règles générales, souvent assises sur des textes juridiques, engageant la responsabilité civile des professionnels et pénale des établissements. Un établissement qui ne satisferait pas aux conditions minimales peut se voir en principe retirer son autorisation. À l'extrême, et même si ces démarches restent isolées, la qualité peut parfois être mise en avant à l'occasion de décisions de fermeture : des activités sont susceptibles d'être abandonnées (maternité, chirurgie), voire des établissements fermés ou reconvertis en fonction de critères de qualité, entendus comme un niveau de sécurité minimal requis pour en maintenir le fonctionnement.

$\mathrm{Au}$ final, la forme de régulation mobilisée correspond selon notre typologie à un régime règlementaire ciblé et à un régime dominant de l'auto-régulation. L'idée est que dans la plupart des cas les professionnels assurent eux-mêmes le pilotage de la qualité. C'est le souci du travail bien accompli et/ou l'observance d'un devoir qui engagent les professionnels à se soumettre à une procédure d'autoévaluation et à réviser leurs savoirs et leurs pratiques en conséquence. Le système professionnel y concourt largement par le biais notamment de la formation continue, de la pression exercée par les pairs et le développement d'instruments d'amélioration de la qualité. L'accréditation opère comme une force externe, mais qui vise à accompagner ce mouvement. L'intervention de la puissance publique se limite au déploiement de quelques outils « autoritaires » destinés à éliminer l'incompétence et à limiter les risques sécuritaires.

Cette association d'une auto-régulation par les professionnels dominante, complétée par une intervention règlementaire au champ d'action ciblé, a longtemps résumé la situation. La persistance des problèmes de qualité et de sécurité, et le constat croissant d'inégalités entre les prises en charge assumées au sein des établissements ont pourtant remis en question cette approche. L'idée que la régulation repose uniquement sur le changement comportemental volontaire des professionnels de santé a perdu du terrain, tandis que le recours à des leviers régulateurs externes conçus pour améliorer la performance a bénéficié d'un intérêt croissant (Institute Of Medicine, 2001) $)^{14}$.

\section{L'introduction des indicateurs : l'oppor- tunité de bâtir une régulation plus externe}

À partir des années 2000, de nouveaux instruments, les indicateurs de mesure de la qualité, ont permis aux pouvoirs publics d'investir justement deux nouveaux modes de régulation, la diffusion publique de l'information, puis plus récemment, l'incitation financière, souvent nommée rémunération à la performance.

En la matière, les développements en France, relativement tardifs par rapport aux pays anglosaxons, ont d'abord été portés par les médias dans le cadre des palmarès des hôpitaux à partir de la fin des années 1990. Plus récemment, les pouvoirs publics se sont également engagés dans cette voie. Un programme national relatif au déploiement et à la généralisation d'indicateurs globaux de qualité a été développé : le projet Coordination de la mesure pour la performance et l'amélioration de la qualité hospitalière (Compaqh) a été créé. Ce programme coordonné par l'Institut national 
de la santé et de la recherche médicale (Inserm) et soutenu par le ministère de la Santé et la HAS, a pour objectif de développer des indicateurs validés de qualité et de sécurité des soins et d'étudier leur emploi dans une perspective d'aide aux établissements et aux pouvoirs publics (Corriol, Daucourt, Grenier et al., 2008) ${ }^{15}$. Deux autres programmes, Clarté et « Path » (Groene, Skau et Frolich, 2008) ${ }^{16}$ sont venus tour à tour complétés le dispositif.

D'une manière générale, la mesure de la qualité et de la sécurité n'est pas sans poser des difficultés méthodologiques. Outre leurs qualités métrologiques, les indicateurs de qualité doivent concerner des résultats cliniques plutôt que la mise en œuvre de procédures, alors que ces derniers sont connus pour être plus simples à concevoir et moins consommateurs de données à collecter. Ils ne doivent pas non plus se focaliser sur un champ d'activité trop restreint au détriment d'autres aspects de la pratique des équipes médicales. Pour autant leur nombre doit être limité pour des raisons de faisabilité ce qui implique une certaine hiérarchisation entre eux. Enfin, de manière à les rendre opérationnels, les indicateurs doivent, dans la mesure du possible, être intégrés au système d'information hospitalier, tout en veillant à la protection des données individuelles des patients. Autant de contraintes qui à ce jour ne sont pas pleinement surmontées en France.

Malgré ces imperfections, la production d'indicateurs en routine à des fins de comparaison entre établissements ou professionnels est devenue une réalité. Elle a permis de construire deux nouvelles formes de régulation, souvent inspirées d'une conception anglo-saxonne du new public management (Pollit, Boucakert, 2000) ${ }^{17}$.

\subsection{La diffusion publique d'information sur la qualité}

Il en est ainsi de la diffusion publique de l'information, constituée à travers la diffusion des classements hospitaliers. Le ministère de la Santé a ainsi mis en place depuis 2006 un tableau de bord fondé sur une batterie d'indicateurs relatifs à la lutte contre les infections nosocomiales dans les établissements de santé, nommé indice composite d'actions de lutte contre les infections nosocomiales (Icalin). Chacun des établissements de santé publics ou privés sur le territoire métropolitain fait ainsi l'objet d'un classement de $\mathrm{A}$ (très bon) à $\mathrm{E}$ (insuffisant). La mise en ligne du site internet «Platines » constitue également une avancée. Bien qu'il ne soit pas focalisé sur la qualité, le site représente une première tentative de recueil synthétique d'une information déjà disponible, rendue publique et permettant une certaine comparaison entre établissements comparables en termes de taille et de statut juridique. Cette première version doit être enrichie des différents indicateurs de qualité à venir. Enfin, à partir de 2009 la procédure de certification des établissements coordonnée par la Haute Autorité de Santé introduit onze indicateurs médicaux relatifs au dossier du patient et à la prise en charge de l'infarctus du myocarde après la phase aigue.

Dans l'ensemble de ces démarches, le mécanisme de régulation est de rendre publique l'information relative à la performance qualitative des établissements. Sur cette base, il est fait l'hypothèse que 
l'usager se comportera en « consommateur éclairé », choisissant son établissement en fonction des résultats observés, et que la publicité faite sur la qualité incitera le professionnel à s'améliorer. Les études empiriques montrent que pour l'instant l'impact de cette approche semble relativement limité en ce qui concerne les patients, mais non négligeable sur la mobilisation des établissements (Fung, Lim, Mattke et al., 2008) ${ }^{18}$.

\subsection{La rémunération à la performance}

La rémunération à la performance vise pour sa part à inciter financièrement en allouant tout ou partie des ressources en fonction du niveau de performance en matière de qualité évalué à partir d'une série d'indicateurs. Cette démarche semble avoir un impact sur l'amélioration de la qualité (Lindenauer, Remus, Roman et al., 2007) ${ }^{19}$. Sa mise en œuvre, en France, déjà engagée à travers les contrats d'amélioration des pratiques individuelles proposés aux médecins généralistes par l'assurance-maladie, se confronte néanmoins à certaines difficultés : $s$ 'appuyer sur des indicateurs fiables et des systèmes d'information appropriés ; définir si l'on valorise l'effort d'amélioration ou si l'on reconnaît les meilleurs ; considérer le niveau d'incitation adéquat; enfin, tenir compte du contexte de contrainte financière au moment d'établir l'investissement dans ce nouveau mode de rémunération (Bras et Duhamel, 2008) ${ }^{20}$. Ces dispositifs reposent par ailleurs sur l'hypothèse d'un comportement utilitariste des professionnels qui ne va pas sans soulever certaines interrogations sur la prise en compte du professionnalisme et de l'éthique, source de motivation intrinsèque des professionnels.

\subsection{Le renforcement du cadre réglementaire}

Durant la même période, plusieurs dispositifs mis en place par les pouvoirs publics sont venus donner une assise institutionnelle à ces nouvelles formes de régulation de la qualité, ou réaffirmer l'intervention règlementaire. Conformément à la loi du 13 août 2004 relative à l'assurance maladie, chaque professionnel est tenu d'évaluer sa pratique sur un thème de son choix. Certains médecins ou certaines équipes particulièrement exposés au risque professionnel peuvent faire accréditer la qualité de leur pratique sur la base d'une analyse des évènements médicaux indésirables et de ceux considérés comme porteurs de risque, en contrepartie d'une aide à la souscription d'une assurance. Enfin, tous les établissements de santé doivent faire l'objet d'une procédure externe d'évaluation dénommée désormais certification (articles L.6113-3 et 4 et R.6113-12 à 16 du Code de la santé publique). La Haute Autorité de Santé (HAS), chargée de l'élaboration et de la mise en œuvre du dispositif, en rend désormais les résultats publics sur son site internet. De même, elle a introduit un niveau de difficulté plus élevé au cours du temps. Enfin, plus récemment la loi " Hôpital, Patient, Sécurité, Territoire », rend obligatoire la remontée d'information relative à certains indicateurs nationaux.

D'autres dispositions, de nature normative, viennent s'ajouter à ces évaluations : dispositifs de vigilance (notamment relatifs aux médicaments, aux dispositifs médicaux et aux produits sanguins labiles), régimes d'autorisation qui concernent la plupart des activités réalisées en établissement de santé. Ces normes et autorisations, attribuées ou contrôlées sous l'égide des Agences Régionales 
de Santé (ARS), déterminent notamment les moyens humains, l'équipement et l'environnement technique nécessaires au bon déroulement de ces activités. Des règles d'organisation pour la prise en charge, et dans certains cas - entre autres pour le traitement des cancers - des seuils d'activité minimale indispensables contribuent aussi à établir a priori la qualité et la sécurité de ces activités (articles R.6122-1 et suivants et D.61241 et suivants du Code de la santé publique).

En définitive, ces dernières années ont été le théâtre de l'affirmation de modes de régulation externes: soient nouveaux, diffusion publique et incitation financière, soient anciens, avec le développement des dispositifs de contrôle.

\section{4. Épilogue ou prologue?}

À la lumière de ce bilan, les quatre formes théoriques de régulation de la qualité d'un service mentionnées dans la typologie, l'autorégulation, l'intervention règlementaire, l'incitation par la diffusion publique et l'incitation financière, apparaissent en action. La régulation de la qualité des soins hospitaliers est donc multiple. Cette synthèse ne rend d'ailleurs pas entièrement compte de la richesse des instruments sous-jacents à chacun de ces registres de régulation. Nous n'avons par exemple fait qu'allusion au régime juridique des professions de santé, omettant de décrire les actions d'investigation et de poursuite en justice. De même, le développement de la formation continue, et les modalités d'évaluation collective organisées par les sociétés savantes professionnelles, enrichissent le registre de l'auto-régulation par les professionnels.
La lecture dynamique de ce mouvement montre également une évolution de l'auto-régulation vers les autres formes de régulation, externes. La régulation de la qualité est donc aussi évolutive. Dans cette évolution, il convient de comprendre qu'un instrument peut alimenter plusieurs formes de régulation. L'accréditation/certification en offre une bonne illustration. Si l'initiative a bien été prise au début comme un accompagnement des professionnels dans leurs démarches d'autoévaluation, l'affichage public des résultats notamment sous forme d'indicateurs, et l'utilisation des résultats par les agences régionales dans le cadre des contrats d'objectifs et de moyens établis avec les établissements de santé, marquent une évolution vers les registres de la diffusion publique et de l'incitation financière.

Multiple, évolutive, il va de soi que ces conclusions se rapportent à l'univers hospitalier. L'avenir inscrit ce type de raisonnement dans une approche plus large, intégrant les soins de ville, ou une lecture territoriale avec la création des agences régionales de santé. Dans cette perspective plus large, on peut s'interroger sur les développements futurs de la régulation de la qualité. Notamment, quelque soit le champ d'analyse, jusqu'où la régulation de la qualité peut-elle s'affirmer dans un contexte où la notion de régulation est spontanément associée à celle de maîtrise des coûts ? Et quel équilibre peut-il s'envisager à terme entre toutes les formes évoquées de régulation, ou dit d'une autre manière entre contrôle externe et autonomie des professionnels ?

Par ailleurs, il n'est pas aisé de comprendre exactement qui régule la qualité. Ce serait une 
erreur d'associer la régulation de la qualité à la seule Haute Autorité de Santé (ex-ANAES). Le « design » institutionnel s'avère de fait plus complexe, impliquant différents services du ministère et des agences sanitaires. L'action de la Haute Autorité de Santé se situe par ailleurs entre « accompagnement des professionnels dans leurs démarches d'amélioration de la qualité » et « régulation »: l'évaluation des pratiques professionnelles (dans la mesure où les résultats, s'ils sont mauvais, ne sont pas suivis de sanction véritable) relève par exemple plus du registre de l'accompagnement; alors que le fait de rendre publics les résultats de la certification relève plus du registre de la régulation.

\subsection{Une régulation « de » ou « par » la qualité?}

Dès qu'une régulation économique s'applique sur un secteur, imposant une contrainte de maîtrise des coûts, il existe un risque de dégradation de la qualité du service. Il n'y a donc pas de régulation économique sans régulation de la qualité à terme. En ce domaine, le secteur hospitalier ne se distingue pas des autres.

Volume et tarif à l'activité fondent aujourd'hui le système de financement des établissements. La question a été posée de savoir si la mise en place de cette nouvelle modalité de financement ne constitue pas un risque pour la qualité du service rendu aux personnes malades. En effet, la tarification à l'activité n'est pas en soi vertueuse. Plus axée sur la productivité, elle ne conduit pas forcément à la recherche d'une amélioration de la qualité. Elle est même susceptible d'induire différents effets pervers : un risque d'aléa moral en fournissant des soins « sur-cotés » à des patients
« légers », un risque de limitation de l'intensité des soins en particulier pour les patients les plus « lourds », un risque de « sacrifice » de la qualité pour réduire les coûts, enfin, un risque de sélection écartant les patients dont la prise en charge ne serait pas associée à un niveau de rémunération suffisant. Mais d'autres analyses soulignent que l'effet inverse est également possible. La qualité, très liée à l'organisation, étant facteur d'efficience, et l'efficience étant elle-même valorisée par la tarification à l'activité, cette dernière devrait permettre d'obtenir le niveau de qualité souhaitable dans les établissements.

Aucun résultat empirique ne laisse penser que la qualité des soins se soit dégradée par la mise en œuvre de la tarification à l'activité ces dernières années (Bousquet, Lombrail et Guisset, 2006). La question ne saurait pourtant être considérée comme réglée dans un contexte où la contrainte sur les coûts ne se relâche pas. Aussi, l'opportunité de promouvoir de nouvelles formes d'incitations à l'amélioration de la qualité semble se justifier. Dans un secteur où la qualité, et sa dimension de sécurité des soins, sont essentielles, certains en viennent même à formuler l'hypothèse que la qualité devienne le critère dominant par rapport auquel se situe l'arbitrage coût-qualité, reprenant ainsi la théorie de la régulation par la valeur (Porter, 2006) ${ }^{21}$. C'est l'objectif qui transparaît à travers l'emploi de l'expression « régulation par la qualité ».

Le développement d'une concurrence entre établissements, voire entre assureurs, faisant l'hypothèse que la qualité constitue un avantage concurrentiel, pourrait faire le jeu d'une telle régulation. Le marché serait alors l'instance de 
régulation de la qualité au risque d'accroître les inégalités. Cet objectif semble néanmoins difficile à concevoir au regard du contexte français actuel. Même si la privatisation du secteur se développe, la puissance publique y est encore très interventionniste, limitant les mécanismes visant à discriminer par la qualité. Ce dernier n'est d'ailleurs pas un objectif en lui-même s'il conduit à augmenter les inégalités. La discrimination par la qualité pourrait par contre avoir l'avantage d'accélérer certaines restructurations. Par ailleurs, la contrainte qui pèse sur l'évolution des dépenses publiques impose de considérer la régulation économique au moins à part égale avec celle de la qualité. Enfin, l'argument des économies relatives au coût de la non qualité un investissement dans la qualité est générateur d'économies - reste au moins pour l'instant virtuel dans les faits.

S'il paraît donc plus raisonnable de parler d'une régulation de la qualité que par la qualité, la question qui se pose consiste à savoir comment articuler cette régulation et la régulation économique. Deux options sont possibles.

La première consiste à faire gérer l'articulation entre les deux approches par la même institution. La seconde consiste à séparer institutionnellement régulation économique et régulation sur la qualité. C'est ce qui s'est fait par exemple au niveau européen en matière de contrôle aérien (Dumez, Jeunemaître, 1999) 22.Dans la mesure où le système hospitalier, et de soins en général, ne pose pas uniquement des problèmes de qualité de service au sens habituel du terme, mais aussi des problèmes de sécurité, comme dans le contrôle aérien, on peut estimer qu'une séparation institutionnelle est souhaitable, et que le système de régulation devrait donc reposer sur deux piliers : une institution en charge de la régulation économique, et une institution de régulation de la qualité. Cela suppose que la Haute Autorité de Santé soit définitivement affirmée dans ce rôle, au détriment de sa fonction d'accompagnement du monde professionnel : avoir des capacités d'accès à l'information, de développement d'études et recherches, et posséder une cellule d'expertise propre, afin de construire les règles de la régulation de la qualité.

\subsection{Avec ou sans les professionnels?}

Entre les quatre formes de régulation proposées, on perçoit que leur cohabitation présente à l'évidence des risques de conflit. L'opposition auto-régulation/ régulation externe est la plus évidente. Par exemple, une règlementation foisonnante et rigide ou des rétributions financières de la performance peuvent affaiblir le sens de l'autonomie d'autant plus lorsqu'elles sont imposées par des bureaucrates béotiens (Marshall, $2005)^{23}$. Penser la cohérence du tout apparaît dès lors comme un impératif, le morcellement de la régulation coûtant de plus en plus cher, au sens littéral comme au figuré. De multiples dispositifs ont été mis en place ces dernières années au sein des établissements de santé, venant souvent se surajouter les uns aux autres. Cet empilement de mesures n'a pas eu que des effets positifs car il peut être facteur de complexité, et parfois source de cloisonnement supplémentaire au sein des établissements.

Assurer une meilleure cohérence peut se concevoir par la volonté de privilégier une forme par 
rapport aux autres. Pour explorer cette possibilité, il est intéressant de revenir sur le caractère multidimensionnel de la qualité. Quelles que soient les dimensions considérées, l'amélioration de la qualité s'appréhende traditionnellement de deux façons distinctes et complémentaires : celle de la minimisation du risque et celle de l'amélioration de la qualité fondée sur le principe d'excellence. Cette dichotomie n'est pas spécifique aux soins, mais il en reste une illustration parfaite. Des erreurs peuvent survenir lors de la réalisation d'actes de soins, rappelant que la sécurité est une facette essentielle de la qualité. La seconde figure est plus positive : une vision du progrès et de la satisfaction complète la quête de l'exécution sans défaut. Les mérites des différentes formes de régulation semblent devoir différer selon que l'objectif poursuivi est la réalisation sans défaut de la prestation-type ou l'excellence.

Ainsi, si la règlementation peut brider la recherche de l'excellence, elle peut garantir un niveau de qualité minimal. Dans le même esprit, le problème de l'observabilité de la qualité des soins peut aussi justifier l'importance accordée à des mécanismes externes, de type diffusion publique de l'information et incitation financière, moins vulnérables à l'opacité et à la critique.

Par contre, la singularité de cette relation de service qui n'acquiert de qualité que dans l'interaction professionnel-usager peut plaider pour une responsabilisation forte des professionnels et l'auto-régulation. La faible appropriation des indicateurs par les professionnels rappelle d'ailleurs que la doctrine d'usage, qui à une politique de régulation externe associe l'hypothèse d'une pratique d'usage spontanée en lien avec les indicateurs, est fausse (Bérard, Gloanec, Minvielle, 2009) ${ }^{24}$ De même, les établissements ou professionnels ne peuvent pas toujours appliquer à la lettre les règles qui font autorité dans la communauté. Les acteurs ne sauraient accepter un instrument de régulation rigide et réducteur qui ignorerait les circonstances. À cet égard, la complexité managériale de la prise en charge des malades qui ne fait que s'accroître (plus de recours technologiques et d'intervenants dans des délais plus courts) plaide dans ce sens.

Enfin, au-delà de la question de savoir si et comment les incitations affectent le comportement du producteur, il faut aussi s'accorder sur la définition du comportement et s'interroger sur ses déterminants. À cet égard, l'auto-régulation favorise la confiance dont les médecins ont besoin et permet de mieux les responsabiliser dans l'actualisation de leurs savoirs (GuiraudChaumeil, 2008) ${ }^{25}$.

En résumé, on le voit, chaque forme de régulation présente des avantages. Il semble dans ce contexte difficile de privilégier une approche par rapport à une autre. Le recours à chacun se justifie. Dans ce dosage, on navigue entre deux écueils symétriques : assurer une certaine cohérence entre les incitations de type externes (diffusion publique de l'information, incitation financière, intervention règlementaire), et l'auto-régulation par les professionnels; ne pas à l'inverse trop strictement lier l'incitation externe et le pilotage autonome de la performance au risque d'optimisation absurde (Lorino, 2001) ${ }^{26}$. On peut ajouter que la question de l'expression de la qualité est aussi au cœur de cet arbitrage. Les formes de régulation externe visant à repérer des qualités 
minimales ou maximales se situent dans une lecture agrégée de la qualité (c'est-à-dire, il y a suffisamment d'évidence tous sujets confondus pour affirmer un niveau de performance élevé ou faible). L'auto-régulation se situe probablement plus sur une lecture plus fouillée, thème par thème, circonstanciée, proche de la lecture professionnelle quotidienne.

Dans cette quête de cohérence, la lecture dynamique du mouvement peut donner une autre clé de lecture. Les formes récentes de régulation peuvent se traduire par un déplacement du rapport de force vers la puissance publique, au détriment des professionnels. Il est possible d'observer une certaine corrélation entre ce déplacement et l'évolution du contexte social. Auparavant, les usagers attendaient des professionnels qu'ils ne nuisent pas, désormais ils exigent les meilleurs soins. Si l'influence de la montée en charge du consumérisme reste encore une chimère, la médiatisation de certains évènements indésirables entraîne une demande de preuve sur la qualité du service (Schilte, Minvielle, 2008) ${ }^{27}$. Le mouvement plaiderait donc désormais pour une régulation externe.

En même temps, les incitations développées au nom de cette régulation externe ne semblent pas pourtant épuiser le sujet. En matière de sécurité des soins, il semble que les dispositifs de diffusion de l'information publique soient moins préconisés au profit du système dit d' "internal reporting $"$ : les professionnels rendent des compte à une structure indépendante sur tous les évènements survenus jugés indésirables, la dite structure gérant la relation avec le professionnel, et assurant une synthèse globale rendue publique qui conserve l'anonymat de ces mêmes professionnels. Ainsi, la régulation apparaît à nouveau fondée sur une auto-régulation par les professionnels. C'est eux qui engagent l'analyse, mais dans un cadre réglementé, une incitation financière pouvant accompagner la déclaration (par exemple, dans le cas de l'accréditation française, la déclaration des évènements porteurs de risque permet au médecin d'obtenir une réduction de son contrat d'assurance en responsabilité professionnelle). Dans l'amorce de ce nouveau mouvement, la confiance semble à nouveau accordée aux professionnels, seuls à même de prévenir les erreurs par un comportement responsable.

Ce cas suggère que de nouvelles formes de régulation se créent, non pour remplacer les précédentes, mais pour les compléter et les améliorer dans ce qui devient au final, un système très sophistiqué. Plus qu'une surenchère instrumentale dans chaque registre, le bénéfice majeur de la régulation réside alors peut-être davantage dans la mise sous tension permanente, faisant osciller l'équilibre entre les formes d'auto-régulation et celles de la régulation externe, en fonction de la confiance accordée aux professionnels de santé pour résoudre les questions de qualité et de sécurité. 
$\mathrm{N} \cdot \mathrm{O} \cdot \mathrm{T} \cdot \mathrm{E} \cdot \mathrm{S}$

1. Par professionnels, nous entendons tous les acteurs des établissements de santé, directeurs d'hôpitaux, médecins, infirmiers. Cette acceptation large du terme est liée à l'objet de l'article, la régulation, qui conduit à considérer tous les membres d'un même établissement porteurs d'une même logique.

2. Deming W.E. Out of the crisis, Cambridge (Mass.), University Press, 1996.

3. Donabedian A. Explorations in Quality Assessment and Monitoring, vol 1 : The Definition of Quality and Approaches to its assessment, Ann Arbor (Mich), Administration Press, 1980.

4. Par exemple dans l'expérience française de développement des indicateurs de qualité, le ministère de la Santé, la Haute Autorité de Santé et le projet Compaq Hpst ont développé, en 2003 puis 2006, 9 objectifs prioritaires.

5. Duhamel G., Minvielle E. «Évaluer et Améliorer la qualité des soins dans les établissements de santé », in Traité d'économie et de Gestion (Bras, Pouvourville, Tabuteau, Eds) ; Sciences Po Ed. 2009.

6. Kaplan R., Norton, D. The Balanced Scorecard: Translating Strategy into Action, Boston (Mass.), Harvard Business School Press, 1996.

7. Sicotte C. et al. "A conceptula Framework for the analysis of Health Care Organization's performance », Health service management research, 1998, 11: 24-48.

8. Porter M. What Is Value in Health Care? N Engl J Med 2010; 363:2477-2481.

9. Lévêque F. Économie de la règlementation. Paris, 1998.

10. Sappington D.E. « Regulating Service quality : A survey ». Journal of Regulatory Economics. 2005, 27-2: 123-54.
11. Pouthier V. Les mécanismes de régulation de la qualité : Définition d'une typologie générique et application au service hospitalier. Rapport Compaqh, Paris, 2006

12. Matillon (Y.) et Durieux (P.), « De l'évaluation à la qualité en médecine ", dans Matillon (Y.) et Maisonneuve (H.), L'évaluation en santé. De la pratique aux résultats, Paris, Flammarion, coll. « Médecine-Sciences », 2007, 180 p.

13. Minvielle (E.), « De l'usage de concepts gestionnaires dans le champ de la santé. Le cas de la qualité hospitalière », La Revue française de gestion, 29 (146), 2003, pp. 167-190.

14. Institute of Medicine. Crossing the quality chasm : a new health system for the 21 st century. Washington DC.2001

15. Corriol (C.), Daucourt (V.) Grenier (C.), et Minvielle (E.), « COMPAQH : recherche sur le développement des indicateurs après trois ans de recul : enseignements », Revue d'Épidémiologie et de Santé publique, 56, 2008, pp. 179-188.

16. Groene (O.), Skau (J. K. H.) et Frolich (A.), «An International Review of Projects on Hospital Performance Assessment », International Journal for Quality in Health Care, 20 (3), 2008, pp. 162-171.

17. Pollitt C., Bouckaert G. 2000. Public Management Reform - A comparative Analysis, Oxford, Oxford University Press.

18. Fung (C. H.), Lim (Y.-W.), Mattke (S.) et al., "Systematic Review: the Evidence that Publishing Patient care Performance Data Improves Quality of Care », Annals of Internal Medicine, 148 (2), 2008, pp. 111-123.

19. Lindenauer (P. K.), Remus (D.), Roman (S.) et al., «Public Reporting and Pay for Performance in Hospital Quality Improvement », The New England Journal of Medicine, 356, 2007, pp. 486-496.

20. Bras (P. L.) et Duhamel (G.), « Rémunérer les 
médecins selon leurs performances : les enseignements des expériences étrangères », Rapport IGAS RM2008047P, juin 2008, www.ladocumentationfrancaise.fr/

21. Porter M, Teisberg E. Redefining health carecreating Value-Based Competition on results. Boston, 2006.

22. Dumez H., Jeunemaître A. 1999, «Les institutions de la régulation des marchés : étude de quelques modèles de référence », Revue internationale de droit économique. 1: 11-30.

23. Marshall M, Harrisson S. It's about more than money: financial incentives and internal motivations. Quality and Safety in Health Care.2005, 14:4-5.

24. 2009. Bérard E., Gloanec M., Minvielle E. «Usage des indicateurs de qualité en établissement de santé ». Journal d'économie médicale, 27-1: 5-20.

25. Guiraud-Chaumeil B., La formation d'un médecin idéal. 2008, La Presse Médicale, 38: 863-868

26. 2001. Lorino P. Méthodes et Pratiques de la performance. Ed de l'Organisation. $3^{\mathrm{e}}$ édition.

27. 2008, Schilte A, Minvielle E. « Le classement des hôpitaux : une nouvelle manière de rendre des comptes ", in Rendre des comptes : une nouvelle exigence sociétale (Dumez ed.). Ed. Dalloz, pp. 64-83.

28. Le Patient Safety and Improvment Act, adopté aux États-Unis en 2005, ou l'accréditation des professions à risque coordonnée par la HAS se fondent par exemple sur ce principe.

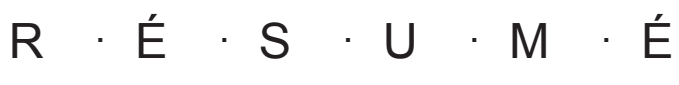

Dans le système de santé, la régulation de la qualité des soins vise à garantir un niveau de qualité égal quelles que soient les conditions d'accès. Fortes de ce principe, les vingt dernières années ont été le théâtre d'un développement important de cette régulation, principalement à l'hôpital. L'objectif de cet article est d'établir une mise en perspective historique de ce développement et d'en déduire une analyse critique. Si cette régulation était peu affirmée dans les années 90 , essentiellement fondée sur des formes d'autoévaluation menées par les professionnels, le début des années 2000 a marqué un virage avec l'émergence de régulations externes (diffusion publique de l'information, paiement à la qualité, renforcement des normes) fondée sur les indicateurs. Sur cette base, nous tentons de préciser le statut de la régulation de la qualité dans des systèmes professionnels de ce type : entre contrôle et autonomie encadrée.

\footnotetext{
Abstract

In health care system, the quality regulation has the aim to guarantee the same level of quality whatever the conditions of access are. Based on this principle, the regulation of quality of care has largely emerged during the last twenty years. The objective of this paper is to describe these new developments. In a first time, this regulation mainly relied on professional peer reviews and auto-evaluation's analyses. At the beginning of the 2000, we can observe new forms of external regulation based on quality indicators (public disclosure, paying for quality) associated to the reinforcement of mandatory rules. On this basis, we try to understand the characteristics of such a type of regulation: between external control and autonomy of the professionals.
} 